\title{
Preparation, Characterization and In Vitro Cytotoxic Activity of Curcuminoid Extract from Wonogiri's Curcuma Xanthorriza RoxB. Loaded Solid Lipid Nanoparticle in Human Breast Cancer
}

\author{
T. Setiawan ${ }^{1 *}$, L. Ambarsari ${ }^{1,3}$ and T. Ibnu Sumaryada ${ }^{2}$ \\ ${ }^{1}$ Department of Biochemistry, Bogor Agricultural University, Indonesia \\ ${ }^{2}$ Department of Physics, Bogor Agricultural University, Indonesia \\ ${ }^{3}$ Tropical Biopharmaca Research Center, Taman Kencana Bogor, Indonesia \\ ${ }^{1}$ tirtasetiawan@apps.ipb.ac.id
}

\begin{abstract}
Wonogiri's Curcuma xanthorriza RoxB., is the medicinal plant that grows in Wonogiri region, contains the highest level of curcuminoid among other regions, and widely used as traditional herbal medicines (Jamu) in Indonesia. Wonogiri's curcuminoid is well known to have wide pharmacological properties particularly in wide range of cancer diseases. However, its water solubility and oral bioavailability find very low. Lipid based carrier called by solid lipid nanoparticles (SLN) may overcome those of limitations. The purpose of this study is to encapsulate Wonogiri's curcuminoid extract into solid lipid nanoparticles (SLN-Cur-Won) to provide prolonged with improve stability bioavailability, involving non-toxic carrier and to observe their cytotoxic activity in vitro. SLN-Cur-Won was prepared by combining both high temperature high-sheer homogenization and ultrasonication techniques. The physiochemical characters were obtained by spectrophotometry UV-Vis, particle size analysis (PS), zeta potential analysis (ZP), polydispersity index analysis (PdI) and entrapment efficiency (EE). In vitro cytotoxic activity through MCF-7 cell lines was determined by using MTT assay. SLN-Cur-Won was found to have maximum entrapment efficiency up to $64.67 \pm 1.843 \%$ and stable at ambient temperature $\left(25^{\circ} \mathrm{C}\right)$ for 1 month. The cytotoxic activity of SLN-Cur-Won in MCF7 cells was four time more potent than the parent Wonogiri's curcuminoid extract (CurWon). Moreover, the SLN exhibited no toxic effects during observations. Wonogiri's curcuminoid extract loaded solid lipid nanoparticles (SLN-Cur-Won) offer promising as potential chemo-preventive agent in breast cancer therapy.
\end{abstract}

Keywords: MTT assay, polydispersity index, solid lipid nanoparticle, Wonogiri's Curcuma xanthorriza RoxB., zeta potential.

\section{Introduction}

Wonogiri's Curcuma xanthorrhiza Roxb., called temulawak by Indonesian people and grouped into the ginger family (Zingiberaceae), is one of the several plants that commonly used in the Indonesian traditional herbal medicines (Jamu). They locally grow in Wonogiri region, Indonesia. The rhizomes of this plant contain rich amount of sesquiterpenes such as xanthorrizol, bisacumol, bisacurol, bisacurone and zingiberene [[1]]. In the previous study, we have found about $75.78 \mathrm{mg} / \mathrm{g}$ of curcuminoid which made Wonogiri's Curcuma xanthorrhiza Roxb as the highest level of curcuminoid compared to the other accessions (Ciemas, Sukabumi, Sragen and Balitro region) in Indonesia [[1],[25]].

Curcuminoid is an orange-yellow polyphenol practically insoluble in water. It is comprised by three main compounds, namely curcumin, demetoxycurcumin and bisdemetoxycurcumin [[2]]. The pharmacological activities of curcuminoid exhibit 
promising antioxidant, anti-inflammatory, antimicrobial and anti-carcinogenic activities. The utilization of curcuminoid as a new natural derivative compound as chemoprevention and cancer chemotherapy has been extensively studied over the past several years in Indonesia. The results of these studies have indicated that curcuminoid play a critical role on multiple molecular targets and have selectively kill tumor cells with low intrinsic toxicity [[3]]. Moreover, other studies have showed that curcuminoid has been considered as a safe supplement due to no toxic effect on normal cells [[4]]. Curcumin, the major contains of curcuminoid, is well known to have anti-carcinogenic activity via interfering the transcription factor $\mathrm{NK}-\mathrm{\kappa B}[[5]]$ and reserves multidrug resistance by downregulating of P-glycoprotein expression in resistant cells [[6]]. However, in spite of the promising anti-carcinogenic activity, the clinical application of curcumin and other its derivative in curcuminoid was limited by extremely low aqueous solubility, rapid metabolism and poor bioavailability [[7]]. To overcome these limitations, some curcuminoid delivery systems (a carrier) have been investigated, including polymer [[8]], cyclodextrin complexation [[9]], chitosome [[10]], liposome [[11]], phospholipid complexes [[12]], nanocrystals [[13]] and solid lipid nanoparticles [[14]].

Solid lipid nanoparticles (SLN), first described by Muller et al. in 1991, noted as an alternative carrier system to traditional emulsion carrier with submicron-sized particles $(50-1000 \mathrm{~nm})$ [[15]]. They involve a physiological and biological properties at both room and body temperature and can be stabilized with nontoxic emulsifiers [[16]]. SLN can be prepared for many different routes of administration including oral, pulmonary, ocular, dermal, parental, and nasal delivery [[17]]. A further study may follow a new drug delivery mode of SLN, apart from direct oral administration. Palmitic acids (PA) are usually used as multifunctional biocompatible and biodegradable excipient found in tissue. In addition, SLN exhibits rapid adsorption and degradation, together with sustained release. All of these characteristics make SLN especially favorable for delivery vehicle. These systems also reduce systemic toxicity, high bioavailability, and rapid absorption followed by rapid onset of action [[18]]. In general, Solid lipid nanoparticles exhibit a novel type of colloidal drug delivery system and offer unique properties such as a small size, a large surface area, and high drug loading [[19]]. Recently studies have been reported that SLN could significantly improve the pharmacokinetic profile and minimize side effects in vivo and in vitro [[20]].

High pressure homogenization, micro-emulsion, solvent emulsion diffusion, solvent emulsification evaporation, high speed stirring and ultra-sonication are some method that commonly used to prepare SLN. Ultra-sonication is widely used technique because of simplicity and effective to production SLN without organic solvent. However, broader particle size distribution ranging up to micrometer and possible to be contamination with metal during sonication addressed to be the limitation of ultra-sonication methods. High sheer homogenization has reported to succesfully produce SLN in nanometer size and also without organic solvent preparations. Ambarsari et al. [[21]] reported that SLN, prepared by using palmitic acid (PA) and poloxamer 188 (PLX) as a surfactant, with average particle size $100-150 \mathrm{~nm}$, were successfully produced by mean of combinations between ultra-sonication and high sheer homogenization at high temperature.

Among types of cancers, breast cancer is the second from several types of carcinomas that commonly incidences of cancer worldwide beside prostate cancer and the second women killer in the world after cervical cancers [[22]]. Despite advances in early detection and standard treatment, breast cancer is often diagnosed at an advanced stage, with 70\% having already given metastases [[23]]. Breast cancer is malignant cancer types, its prognosis is relatively poor and the 5-years survival rate is less than $15 \%$ [[24]]. It is clear that a new approach and a new formulation of drug against this cancer is urgently needed. 
The Study of SLN as a carrier of Wonogiri's curcuminoid extract (Cur-Won) for cancer treatment has not been reported. In addition, the using of palmitic acid (PA) as lipid system of SLN has limited to find. Therefore, in this study we designed a research of Wonogiri's curcuminoid extract loaded SLN (SLN-Cur-Won) as vehicles offering better stability, with nontoxic nano-carrier, and increase the cytotoxicity. In this work, SLNCur-Won was first prepared using combination between ultra-sonication and high sheer homogenization at high temperature. The physicochemical properties were characterized in term of the shape, size, zeta potential, entrapment efficiency and storage stability study. The cytotoxicity was evaluated by 3-(4,5-dimethylthiazol-2-yl)-2,5-diphenyl-tetrazolium bromide (MTT) assay using MCF-7 cell lines (human breast cancer). The work flow of various steps used in this study was showed in Error! Reference source not found..

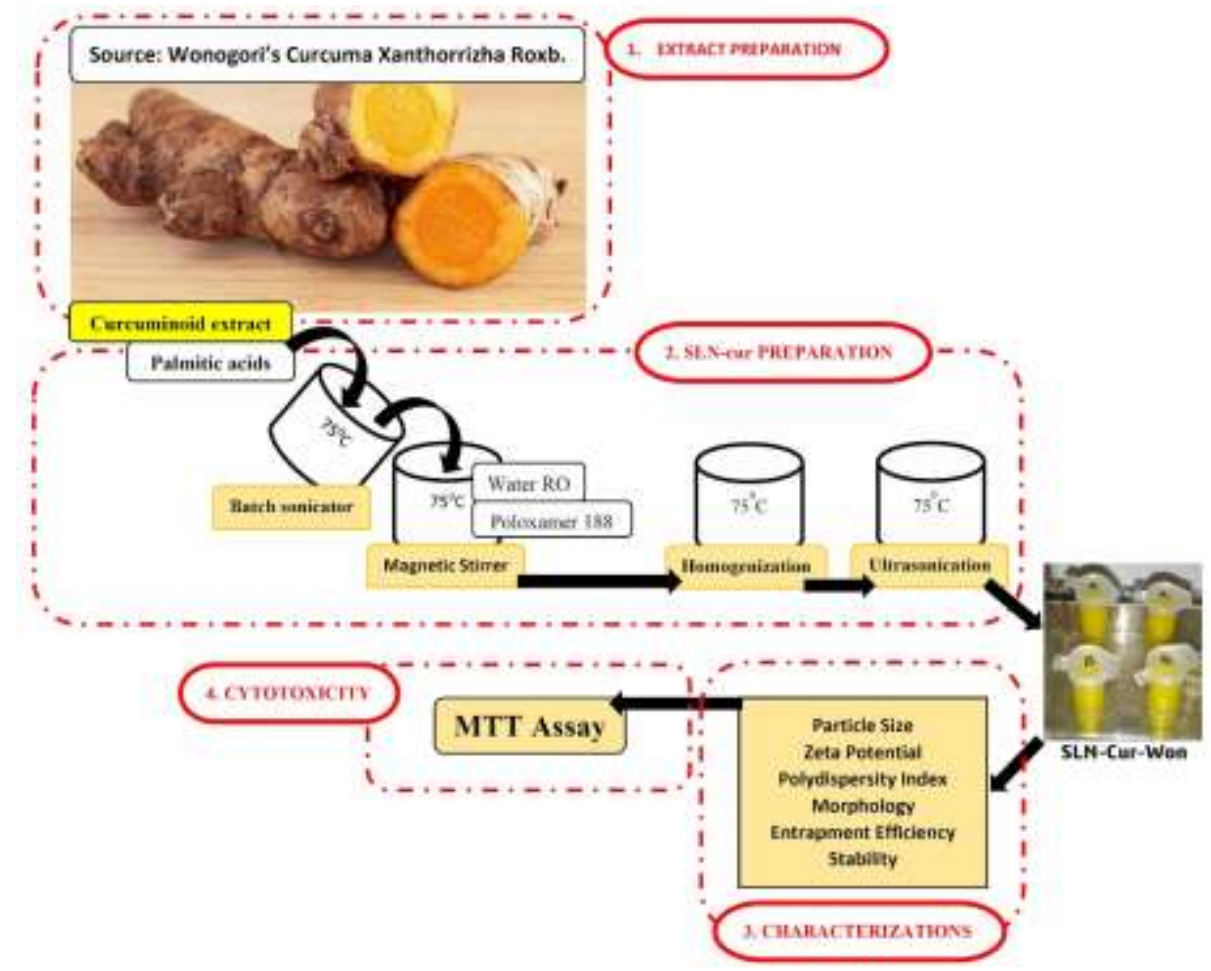

Figure 1. Flow Diagram Depicting Various Step used in this Study

\section{Study Methods}

\subsection{Materials}

The extract Wonogiri's curcuminoid (Cur-Won) was extracted and collected from previous study [[25]]. Curcuminoid standard 94\% (Cur-Std) was obtained from Tropical Biopharmaca Research Centre (PSB), SOHO. Palmitic acid (PA) (Merck), poloxamer 188 (PLX) (BASF) and deionized water, were obtained from Merck. Homogenizer (Ultra Turrax T18), spectrophotometer UV-Vis (Ocean Optic USB4000) and ultrasonic processor (130 Watt $20 \mathrm{kHz}$, Cole-Parmer) were obtained from Biochemistry Laboratory IPB. Particle size analyzer (Delsa NanoC, Beckman Coulter) was obtained from Physics Laboratory IPB and Nano-center Serpong. Micro-centrifuge (MIKRO 200R, Hettich Zentrifugen) was obtained from microbiology laboratory of Primate Research Centre, Indonesia. The MCF-7 (human breast cancer cells), RPMI 1640, FBS (fetal bovine serum), MTT reagent, sodium carbonate, DMSO 100\%, fungison, trypsin EDTA, 
penicillin streptomycin, trypan-blue, reagent stopper, ELISA reader, alcohol and PBS were obtained from Primate Research Centre (PSSB) culture collection, Indonesia.

\subsection{Spectrophotometer UV-Vis Analysis}

A number of curcuminoids inside the nanoparticle was quantified using spectrophotometer UV-Vis methods. Briefly, the maximum absorbance of Cur-Std was measured. A calibration curve with acceptable linearity $\left(R^{2}=0.9999\right)$ was constructed by plotting the peak area versus Cur-Std concentration within the range $0.001-0.006$ $\mathrm{mg} / \mathrm{mL}$ and detected at a wavelength of $425 \mathrm{~nm}$ (maximum absorbance).

\subsection{Preparation of SLN-Cur-Won, SLN-Cur-Std and SLN}

SLN-Cur-Won and SLN-Cur-Std were prepared using homogenization-ultra sonication method as suggested by Ambarsari et al. [[21]]. The PA; Cur-Won; PLX with weight ratio 1: 0.5: 1.5 was stated as optimal result to produce SLN-Cur-Won. Briefly, the lipid phase consisting of PA and Cur-Won was heated to $75^{\circ} \mathrm{C}$. The aqueous phase was prepared by dissolving PLX in $100 \mathrm{~mL}$ deionized water and heated to $75^{\circ} \mathrm{C}$. Then, lipid phase was added to aqueous phase and stirred for 5 min following which homogenized at 13.500 $\mathrm{rpm}$ for $1 \mathrm{~min}$. Emulsion, thus obtained, was sonicated for $2 \mathrm{~h}$.

SLN-Cur-Std was generated by using similar method before, the difference is that CurWon was changed by Cur-Std. SLN is comprised by lipid (PA) and surfactant (PLX). In general, SLN was prepared by using similar procedure as preparing SLN-Cur-Won. The difference is that SLN was prepared without any added compounds. Since SLN-Cur-Won, SLN-Cur-Std and SLN were completely prepared, they were stored at $4^{0} \mathrm{C}$ in refrigerator and protected from light until further use.

\subsection{Particle Size and Zeta Potential Analysis}

The particle size (PS), size distribution (PdI) and zeta potential (ZP) of nanoparticle were determined by dynamic light scattering (DLS) method using a Delsa Nanosizer. The intensity of scattered light was fixed at $90^{\circ} \mathrm{C}$ to an incident beam. Prior to measurement, both SLN-Cur-Won, SLN-Cur-Std and SLN suspensions were diluted with Mili-Q water to suitable concentrations in an effort to obtain better focusing. All analyses were performed in the auto-measuring mode at $25^{\circ} \mathrm{C}$, and the results were presented as the average value of triplicate sampling and measurement for each formulation [[21]].

\subsection{Morphology of the Nanoparticles}

The morphology of SLN-Cur-Won, SLN-Cur-Std and SLN were characterized by mean of a transmission electron microscope (TEM) [26]. Briefly, a drop of the nanoparticle suspension was placed on a membrane grid surface with a filter paper. Phosphotungstic acid $(2 \% \mathrm{w} / \mathrm{v})$ was used for negative staining. The excess fluid was removed from the carbon coated grid. Then after, grid surface was air dried before loaded onto the microscope.

\subsection{Entrapment Efficiency (EE)}

The SLN-Cur-Won and SLN-Cur-Std were centrifuged at $14.000 \mathrm{rpm}(18.626 \mathrm{x} \mathrm{G})$ at $4^{0} \mathrm{C}$ for $40 \mathrm{~min}$ and supernatant was decanted. The pellet was macerated by methanol to extract the curcuminoid from SLN and then re-centrifuged. The supernatant absorption was measured by spectrophotometer UV-Vis at $425 \mathrm{~nm}$. The EE was calculated by using equation below [[21]]:

$$
\text { Entrapment efficiency }(\%)=\frac{[\text { Entrapped curcuminoids }]}{[\text { Total curcuminoids }]} \times 100 \%
$$




\subsection{Stability Studies of SLN-Cur-Won}

The SLN-Cur-Won was tested for their stability [[28]]. Freshly prepared SLN-CurWon in three samples were sealed in vials and placed in a stability chamber maintained at $25^{0} \mathrm{C}$ with sampling frequency of one week $(n=3)$. Sealed SLN-Cur-Won in stability chamber was analyzed over a month for PS, ZP, PdI and EE.

\subsection{MTT Assay}

The MCF-7 human breast cancer cells were obtained from Primate Research Centre culture collection (PSSP, Bogor, Indonesia). The cells were grown in Roswell Park Memorial Institute (RPMI)-1640 medium supplemented with $10 \%$ fetal bovine serum, a mixed solution of 100 units $\mu \mathrm{g} / \mathrm{ml}$ penicillin and $100 \mu \mathrm{g} / \mathrm{ml}$ streptomycin (Gibco BRL) and incubated in a humidified incubator at $37^{\circ} \mathrm{C}$ with $5 \% \mathrm{CO}_{2} / 95 \%$ air. Cellular growth inhibition/cytotoxicity was determined in MCF-7 cell line using a MTT assay [32]. Standard curcuminoid purity 94\% SOHO (Cur-Std), Wonogiri's curcuminoid extract (Cur-Won), SLN, SLN-Cur-Std and SLN-Cur-Won were freshly prepared at 4 various concentrations of $62.5,31.25,15.62,7.81 \mu \mathrm{g} / \mathrm{ml}$. The cells were digested and seeded in 96-well plate at a density of $5.0 \times 10^{4}$ cells with $100 \mu \mathrm{l} /$ well for $24 \mathrm{~h}$. Cur-Std and CurWon were dissolved in dimethyl sulfoxide (DMSO) and then diluted in RPMI-1640 medium to obtain a final concentration of DMSO $<0.2 \%$. The SLN-Cur-Std, SLN-CurWon and SLN were directly dissolved and diluted with RPMI-1640 medium. The final concentration of DMSO in the culture medium was below $0.25 \%$. After standing for 24 $\mathrm{h}$, medium was removed and MTT assay was performed. Each well was filled with $500 \mu \mathrm{l}$ of containing of MTT-reagent consisting of $5 \mathrm{mg} / \mathrm{ml}$ MTT in PBS. After incubation for 3 $\mathrm{h}$ at $37^{\circ} \mathrm{C}$, medium was removed and $500 \mu \mathrm{l}$ stop-solution (10\% SDS and 50\% dimethyl formamide was added. The cells were incubated overnight at $37^{\circ} \mathrm{C}$ and absorbance was measure using an Elisa reader at $595 \mathrm{~nm}$. All experiments were performed in triplicates.

\subsection{Statistical Analysis}

The results were presented as the mean \pm SD. The statistical analysis of samples was performed by using a Student's t-test with $p$-values $<0.05$ as minimal level significance.

\section{Results}

\subsection{Characteristics of Nanoparticles}

The Table 1 shows the details regarding the physicochemical properties in three groups of nanoparticles. SLN, the lipid compartement only comprised by PA and PLX, has the smallest physicochemical values among three groups of nanoparticles and the only one that has not EE value. It was tested to have $301.05 \pm 5.4 \mathrm{~nm}$ of PA, $31.3 \pm 1.5-\mathrm{mV}$ of ZP and $0.2180 \pm 0.002$ of PdI. SLN-Cur-Std is the second smallest, in which it was found to be $316.17 \pm 0.3 \mathrm{~nm}, 32.2 \pm 0.9-\mathrm{mV}, 0.2775 \pm 0.005$ of PS, ZP and PdI, respectively. Following which, SLN-Cur-Won is the largest, it was noted to have $354.52 \pm 3.6 \mathrm{~nm}, 33.6$ $\pm 1.2-\mathrm{mV}$, and $0.3101 \pm 0.012$ of PS, ZP and PdI, respectively. In summary, the overall nanoparticles physicochemical properties showed similar. The PS is ranged approximately between $300-350 \mathrm{~nm}$, ZP values exhibit higher than $-30 \mathrm{mV}$, PdI was noted by lesser 0.32 . In term of EE, SLN-Cur-Std, for certain, shows $5.86 \%$ better than SLN-Cur-Won. 
Table 1. The Physicochemical Properties

\begin{tabular}{lcccc}
\hline Groups & PS (nm) & ZP (-mV) & PdI & EE (\%) \\
\hline SLN-Cur-Won & $354.52 \pm 3.6$ & $33.6 \pm 1.2$ & $0.3101 \pm 0.012$ & $64.67 \pm 1.843$ \\
\hline SLN-Cur-Std & $316.17 \pm 0.3$ & $32.2 \pm 0.9$ & $0.2775 \pm 0.005$ & $70.13 \pm 0.965$ \\
\hline SLNs & $301.05 \pm 5.4$ & $31.3 \pm 1.5$ & $0.2180 \pm 0.002$ & - \\
\hline Note: Results are presented as mean \pm standard deviation (S.D.), $\mathrm{n}=3$. &
\end{tabular}

\subsection{Morphology of Nanoparticles}

Error! Reference source not found. shows the photograph of the color differences between SLN, and SLN-Cur-Won. The SLN (Error! Reference source not found.a) completely white. Conversely, SLN-Cur-Won (Error! Reference source not found.c) has yellow color indicated that SLN absorbs the Cur-Won (Error! Reference source not found.b). The TEM micrographs also showed that the morphologies among SLN-Cur-Std (Error! Reference source not found.a), SLN-Cur-Won (Error! Reference source not found.b) and SLN (Error! Reference source not found.3c), were nearly rounded spherical in shape with clear and smooth surface. Most of the particles were approximately distribute with size ranging 150 to $350 \mathrm{~nm}$ under TEM.
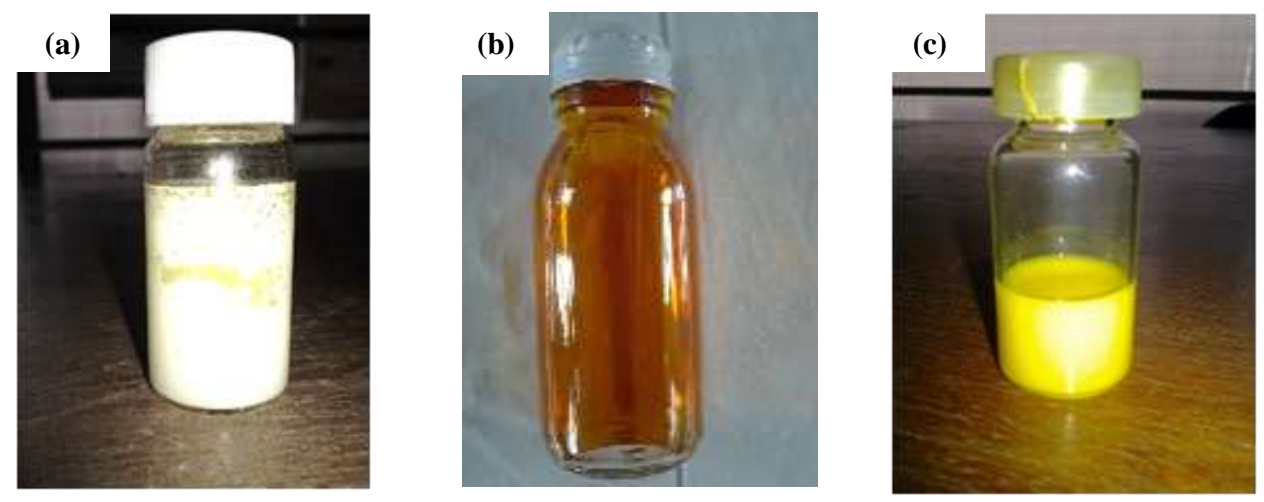

Figure 2. Photographs Showing. (a) SLN; (b) Cur-Won; (c) SLN-Cur-Won
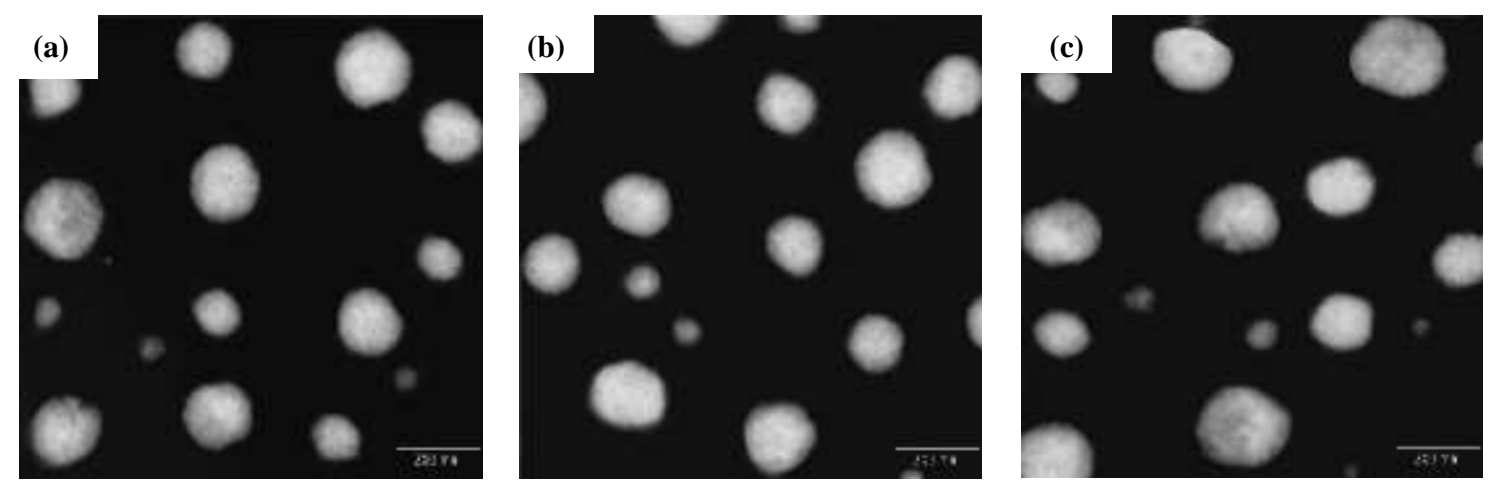

Figure 3. Photographs showing transmission electron microscope (TEM). (a) SLN-Cur-Std; (b) SLN-Cur-Won; (c) SLN

\subsection{Stability Study of SLN-Cur-Won}


Error! Reference source not found. shows that there is a gradual decrease of EE for SLN-Cur-Won over a month. This percentage gradually declines approximately $1-2 \%$ a week. Conversely, Error! Reference source not found. also shows a gradual increase of the PS, PdI and ZP of SLN-Cur-Won over a month. The PS of SLN-Cur-Won was continuously change by higher than 3-4 nm per week. Following which, the PdI value increases frequently by $0.011-0.016$ every week. The ZP also shows declines slightly till the beginning of 1 week and increase steadily by -0.45 to $-0.49 \mathrm{mV}$ per week on the next week. Nearly similar pattern was observed to all of stability parameter at the last two weeks. The values remain stable and change consistently with a small number of differences. Statistical calculation revealed that there has significant differences regarding the test periods on parameters. These results indicate that SLN-Cur-Won expected to be changes frequently over a month in ambient temperature.

Table 2. Stability Study of SLN-Cur-Won over 1 Month

\begin{tabular}{lccccc}
\hline \multirow{2}{*}{$\begin{array}{c}\text { Stability } \\
\text { parameters }\end{array}$} & \multicolumn{5}{c}{ Test Periods } \\
\cline { 2 - 6 } & 0 week & 1 week & 2 week & 3 week & 4 week \\
\hline $\mathrm{PS}(\mathrm{nm}) * * *$ & $356.32 \pm 2.1$ & $359.6 \pm 3.6$ & $364.6 \pm 4.4$ & $368.77 \pm 1.6$ & $368.82 \pm 0.1$ \\
\hline $\mathrm{EE}(\%)^{* * *}$ & $64.3 \pm 0.2$ & $63.6 \pm 1.3$ & $62.2 \pm 1.2$ & $61.0 \pm 0.4$ & $60.98 \pm 0.6$ \\
\hline $\mathrm{PdI} * * *$ & $0.331 \pm 0.07$ & $0.342 \pm 0.14$ & $0.358 \pm 0.69$ & $0.371 \pm 0.09$ & $0.375 \pm 0.12$ \\
\hline $\mathrm{ZP}(-\mathrm{mV})^{* * *}$ & $34.6 \pm 2.5$ & $33.97 \pm 1.1$ & $34.42 \pm 4.6$ & $34.87 \pm 3.21$ & $34.88 \pm 1.43$ \\
\hline
\end{tabular}

Note: Results are presented as mean \pm standard deviation (S.D.), $\mathrm{n}=3$ (*** $p<0.001$, Student's t-test).

\subsection{Cytotoxicity Assay}

The bar chart on Error! Reference source not found. indicates a gradual decrease of MCF-7 cell viabilities on four different concentration groups on the percentages contributing to their cytotoxic activities. These percentages are divided into have effect (less than 100\%) and have no effect (100\% and above). Following which the concentration groups are comprised by Cur-Std, Cur-Won, SLN, SLN-Cur-Std and SLNCur-Won. On the smallest concentrations, Cur-Won and SLN were the only treatment has no effect in MCF-7 viability. In the other hand, Cur-Std showed moderate effect in cell viability among the groups. Conversely, SLN-Cur-Std exhibited the best effect with nearly left only $30 \%$ survival cells followed by SLN-Cur-Won which left nearly a half survival MCF-7 cells. On the second highest concentrations, SLN groups were the only treatment that showed above $100 \%$ cell viabilities and expected to have no significant effect on the highest concentrations. In the other hand, Cur-Won started the initial effect after killing about $10 \%$ cells. The survival cells treated by Cur-Std, SLN-Cur-Std and SlnCur-Won in this concentrations began declining a half than before. On the second highest concentration Cur-Won has left nearly about $2 \%$ higher survival cells than Cur-Std. SLNCur-Won observed has similar effect on this concentration to SLN-Cur-Std, left approximately $10 \%$ survival cells. On the last concentration, less than $1 \%$ cells survive from all treatments except SLN.

Table 3. The $\mathrm{IC}_{50}$ Values

\begin{tabular}{cccccc}
\hline Groups & $\begin{array}{c}\text { Cur-std } \\
(\mu \mathrm{g} / \mathrm{ml})\end{array}$ & $\begin{array}{c}\text { Cur-Won } \\
(\mu \mathrm{g} / \mathrm{ml})\end{array}$ & $\begin{array}{c}\text { SLN-Cur-std } \\
(\mu \mathrm{g} / \mathrm{ml})\end{array}$ & $\begin{array}{c}\text { SLN-Cur-Won } \\
(\mu \mathrm{g} / \mathrm{ml})\end{array}$ & $\begin{array}{c}\text { SLN } \\
(\mu \mathrm{g} / \mathrm{ml})\end{array}$ \\
\hline $\mathrm{IC}_{50}$ & $8.796 \pm 0.792$ & $25.21 \pm 0.728$ & $1.39 \pm 0.138$ & $6.04 \pm 2.309$ & $254.3 \pm 8.008$ \\
\hline Note: Results are presented as mean \pm standard deviation (S.D.), $\mathrm{n}=3$. & &
\end{tabular}




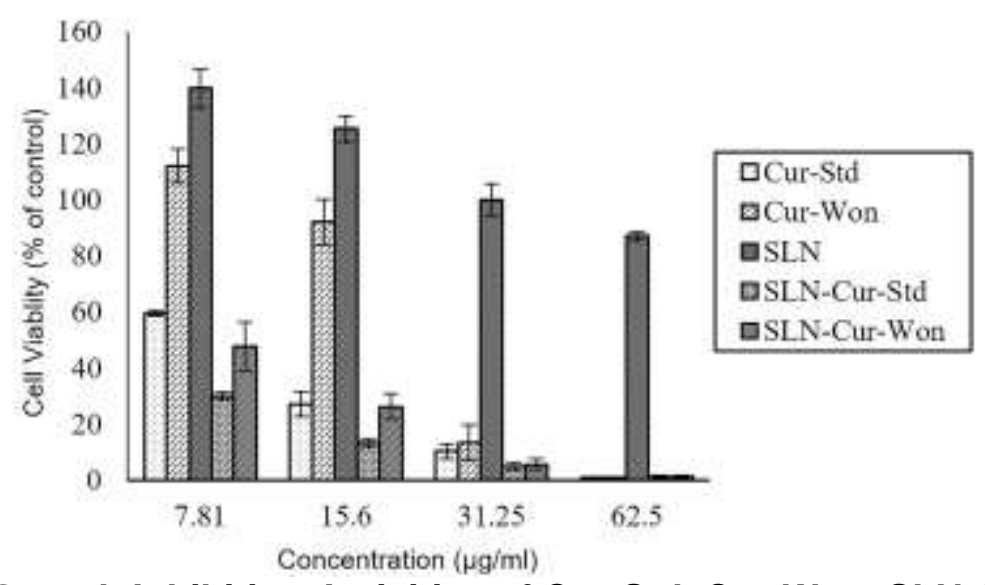

Figure 4. Growth Inhibition Activities of Cur-Std, Cur-Won, SLN-Cur-Std, SLN-Cur-Won and SLN on MCF-7 cells after $24 \mathrm{~h}$ Incubation $(n=3)$. Results are Presented as Mean \pm standard deviation (S.D.)

\section{Discussion}

In our previous study, we have successfully retrieved various curcuminoid extract from different accessions and tested their cytotoxicity in vitro. The results showed that curcuminoid extract from Wonogiri accession has possibility of selecting high quality clone curcuminoid production, approximately $75,78 \mathrm{mg} / \mathrm{g}$. Moreover, they showed anticancer activity in vitro in varies dose dependent [[1],[25]]. The advances of promising therapeutic efficacy of curcuminoid such as Wonogiri's curcuminoid against cancer always get blocked due to their certain limitations for instances, low oral bioavailability and rapid metabolism [[7]]. The encapsulation curcuminoid into lipid-based nanoparticles has been reported to overcome those of limitations [[11]]. The certain encapsulation of Wonogiri's curcuminoid inside the SLN in amorphous or molecularly dispersed was confirmed in the previous study by using NMR and DSC analysis [[21]]. However, in vitro and in vivo data are still limited. Therefore, in this study we design the encapsulated Wonogiri's curcuminoid extract into solid lipid nanoparticles (SLN-Cur-Won) to provide prolonged with improve of curcuminoid stability and bioavailability, involving non-toxic carrier, and observe their cytotoxic activity (in vitro).

The main techniques to prepare SLN are high sheer homogenization and ultrasonication [[26]]. Compared to other techniques such as micro-emulsion, high pressure homogenization, solvent emulsion diffusion, solvent emulsion evaporation, and high speed stirring, ultra-sonication technique is widely used due to simplicity of method and effective to production SLN without organic solvent. However, broader particle size distributions ranging into micrometer and metal contaminations are addressed to be a big problem of this technique. Thus, In this study we combine between high sheer homogenization with high temperature and ultra-sonication, to elicit those problems [[21]].

The first physio-properties to alter SLN-Cur-Won and SLN are by their color. SLN was clearly white in color (Error! Reference source not found.a). The SLN-Cur-Won was brightly in yellow color (Error! Reference source not found.c) due to the adding of curcuminoid (orange-yellow color properties) during preparations (Error! Reference source not found.c). The PS of SLN in our study was found to be $301.05 \pm 5.4 \mathrm{~nm}$. However, the particle size of SLN-Cur-Won increased slightly to $354.52 \pm 3.6 \mathrm{~nm}$ since adding of Cur-Won. The increases of particle size of SLN-cur were due to the drug pay load Cur-Wont in the lipid core [[27]].

Lipid nanoparticles were famously able to be homogenously dispersed into water. Curcuminoid which has hydrophobic characteristics can be predicted to be able to absorb into lipid core rounded spherical in shape. Transmission electron microscope (TEM) 
revealed that SLN, SLN-Cur-Std and SLN-Cur-Won were successfully homogenous without any apparent drug crystals. The TEM image of SLN, SLN-Cur-Std and SLN-CurWon suspension had a regular spherical shape with clear, smooth surface and no adhesion on it (Error! Reference source not found.). The PLX, non-ionic co-surfactant, played a critical role in the stability of lipid nanoparticle system. High surfactant concentrations effectively stabilize the particle created by forming a steric barrier on the particle surface, aimed to protect the particles from coagulation and to make spherical in shape and also improve the EE [[27]-38].

The PdI is one of the most important properties used to evaluate the stability of colloidal system [[28]]. The polydispersity index (PdI) is also an indicator of homogeneity of the size distribution, and narrow size distribution and uniform size confirmed the homogenous nature of the formulation. The PdI of SLN, SLN-Cur-Std and SLN-Cur-Won also showed in approximately 0.3 values (Error! Reference source not found.) indicates that size of particle in the emulsion is relatively similar in size (homogeny) [[28]-31]. As a PdI, ZP is another important parameter to determine the stability of colloidal dispersion. The $\mathrm{ZP}$ is value that represents the electrostatic surface of SLN. The electrostatic repulsion among nanoparticles can prevent the coalescence of droplets [[28]]. The value of \pm 30 was considering to be acceptable producing stable colloidal systems [[29]]. In our study, the absolute value of ZP of SLN, SLN-Cur-Std and SLN-Cur-Won were approximately about $-30 \mathrm{mV}$ (Error! Reference source not found.). Therefore, they demonstrated that the colloidal dispersion is a physically stable in the emulsion. Following which, the absolute ZP in our study was found negative. The negative charge observed is due to the released fatty acid from the hydrolysis PA during preparations. In that condition, the hydrophilic emulsifiers were thought to align alongside each other, making more rigidity and strength to the emulsifier over hydrogen bonding [30]. The PLX acted as a non-ionic surfactant tends to reduce the absolute value of ZP [31]. Although PLX is classified as a nonionic polymer, its molecules contain besides hydroxyl $\left(\mathrm{OH}^{-}\right)$ groups some Hydrogen $\left(\mathrm{H}^{+}\right)$ones. Thus, the $\mathrm{C}-\mathrm{H}$ bonds in $\alpha$ position in relation to have acidic properties. Therefore, in this way the molecules gain negative charge then results in more negative ZP [[29]]

The amount of drug to be incorporated into the delivery system is dependent on the physicochemical properties of drug. Despite the fact that high EE was the result of high solubility of the drug in melted lipid, the surfactant also possessed similar importance [[30]3]. Based on PA properties, curcuminoid may be more likely distributed in the melted lipid core of nanoparticle because it contains a hydrophobic chain. In our study, the EE of SLN-Cur-Won was calculated and the results was found to be $64.67 \pm 1.843 \%$. This result indicated that Cur-Won was successfully added in the core of SLN. Conversely, the EE of SLN-Cur-Std showed better than SLN-Cur-Won. It may be that Cur-Std has better purity than Cur-Won. The purest curcuminoid (Cur-Std) made it easier to incorporated in the melted lipid core than Cur-Won. In addition, previous reports suggested that the SLN formulation stabilized by $1 \%$ non-ionic co-surfactant are acceptable for the incorporation of lipophilic drugs [[36]-37].

Stability study of SLN-Cur-Won were designed to investigated the storage stability of lyophilized over a prolonged period of time. As previously indicated from PdI and ZP measurement, SLN-Cur-Won was expected stable, in term in the short time. In order to assess the effect of the storage temperature stability in term in the long time, SLN-CurWon was investigated with regard to their physiochemical stability for a month in ambient temperature $\left(25^{\circ} \mathrm{C}\right)$. Despite the fact that in our experiment the stability parameters changed significantly $(p<0.05)$ on storage up to a month (Error! Reference source not found.); however, the nanoparticles indicated stable. It means that our method is suitable for the production of stable SLN-Cur-Won. The good stability may because of the slow transition/destruction of lipid in the SLN lead to restrain the extract settled in the core [[34]-36]. The change values may due to the disrupting of the surfactant (PLX) during 
observation led to weaken internal lipid binding. Since the amount of drug loads, ZP and PS are dependent on lipid-surfactant ratio, a decrease in content of the surfactant led to the increases of PS, ZP, PdI, and decreases of drug load (EE) [32-39].

In order to study the cytotoxicity, MTT assay was performed on human MCF-7 cells for $24 \mathrm{~h}$ incubation (Error! Reference source not found.). The cellular viabilities of MCF-7 cells incubated with SLN suspension up to the highest concentration were in the range $87-136 \%$ (Error! Reference source not found.), indicated that the cells still capable to survive in those concentrations. This finding also proposed that SLN (PA and PLX) in SLN-Cur-Won, have no effect on cell viability of MCF-7. The inhibition efficiency of Cur-Won increased slightly when encapsulated in SLN. Base on $\mathrm{IC}_{50}$ value (Error! Reference source not found.), SLN-Cur-Won displayed four times more potent than Cur-Won (Error! Reference source not found.).

The findings of the present study prove that SLN-Cur-Won is better internalized into MCF-7 cells. Some studies using curcumin extract also claimed that curcumin loaded SLN was more effective anti-proliferative while compared to curcumin [[37]-37]. The particle size and the surface charge on the SLN may be the reason for better cell uptake of curcuminoid in the form of SLN-Cur-Won.

\section{Conclusion}

The SLN-Cur-Won prepared by combination of high sheer homogenization with high temperature and ultra-sonication shows stable at ambient temperature $\left(25^{\circ} \mathrm{C}\right)$ for a month at $64.67 \pm 1.843 \%$ of maximum entrapment efficiency and achieves better cytotoxicity in lesser dose compared to Cur-Std and Cur-Won. SLN-Cur-Won showed four times more potent than Cur-Won and $2.756(\mu \mathrm{g} / \mathrm{ml})$ better than CurStd. following which, the SLN, comprised by palmitic acid and PLX, exhibits no toxic effect during the study. Therefore, this study proposed that SLN offer promising delivery system to increase Cur-Won cytotoxic activity in MCF-7 cells and could be exploited as potential chemo-preventive agent in breast cancer therapy.

\section{Acknowledgments}

The research thanks to Directorate General of Higher Education, Republic of Indonesia (DIKTI) and Bogor Agricultural University. This research was partially funded by Competitive Research Grant from Directorate of Higher Education, Ministry of Education and Culture Indonesia (RAPID) program with contract number: 083/SP2H/PL/ Dit.Litabmas/II/2015.

\section{References}

[1] W. Nurcholis, L. Ambarsari, E. J. Purwakusumah, "Curcumin analysis and cytotoxicity activities of some Curcuma xanthorrhiza Roxb. Accessions Int”, J. Pharm. Tech., Vol. 9, (2016), No. 7, pp. 175180.

[2] B. B. Aggarwal, I. D. Bhatt, H. Ichikawa, K. S. Ahn, G. Sethi, "Turmeric: The Genus Curcuma”, Taylor and Francis, New York, (2006), pp. 298-303.

[3] J. Ravindran, S. Prasad, B. B. Aggarwal, "Curcumin and Cancer cells: How many ways can curry kill tumor cell selectively”, AAPS. J., Vol. 11, (2009), pp. 495.

[4] B. B. Aggarwal, A. Kumar, A. Bharti, "Anticancer potential of curcumin: preclinical and clinical studies", Anticancer Res, Vol. 23, (2003), pp. 363-398.

[5] B. H. Choi, C. G. Kim, Y. Lim, Y. H. Lee," Curcumin down-regulates the multidrug-resistance mdr1b gene by inhibiting the PI3K/Akt/NFkB pathway", J. can. Let., Vol. 224, (2008), pp. 111.

[6] W. Punfa, S. Suzuki, P. Pitchakarn, S. Yodkeeree, T. Naik, S. Takahashi, P. Limtrakul, "Curcuminloaded PLGA nanoparticles conjugated with anti-P-glycoprotein antibody to overcome multidrug resistance", Asian Pas. J. Canc. Prev., Vol. 15, (2014), pp. 9249-9258. 
[7] Q. Tan, J. Wu,Y. Li, H. Mei, C. Zhao, J. Zhang,"A super-molecular curcumin for enhanced antiproliferative and pro-apoptotic activities: molecular characteristics, computer modeling and in vivo pharmacokinetics", Nanotechnology., Vol. 13, (2013), pp. 35102.

[8] P. Zou, L. Helson, A. Maitra, S. T. Stern, S. E. McNeil, "Polymeric Curcumin Nanoparticle Pharmacokinetic and Metabolism in Bile Duct Cannulated Rats", Mol. Pharm., (2013).

[9] P. A. Patel, S. C. Patil, D. R. Kalaria, Y. N. Kalia, V. B. Patravale, "Comparative in vitro and in vivo evaluation of lipid based nano-carriers of Huperzine A", Int. J. Pharm., Vol. 446, (2013), pp. 16-23.

[10] J. Zhang, Q. Tang, X. Xu, N. Li, "Development and evaluation of a novel phytosome-loaded chitosan microsphere system for curcumin delivery", Int. J. Pharm., Vol. 448, (2013), pp. 168-174.

[11] L. Li, F. S. Braiteh, R. Kurzrock, "Liposome-encapsulated curcumin: in vitro and in vivo effects on proliferation, apoptosis, signaling, and angiogenesis", Cancer., Vol. 104, (2005), pp. 1322.

[12] K. Maiti, K. Mukherjee, A. Gantait, B. P. Saha, P. K. Mukherjee, "Curcumin-phospholipid complex: preparation, therapeutic evaluation and pharmacokinetic study in rats", Int. J. Pharm., Vol. 330, (2007), pp. 155.

[13] H. Rachmawati, I. Al Shaal, R. H. Muller, C. M. Keck, "Development of curcumin nanocrystal: physics aspects", J. Pharm. Sci., Vol. 102, (2013), pp. 204.

[14] P. Fadda, M. Monduzzi, F. Caboi, S. Piras, P. Lazzari, "Solid lipid nanoparticle preparation by a warm micro-emulsion based process: Influence of micro-emulsion microstructure", Int. J. Pharm., Vol. 446, (2013), pp. 166-175.

[15] R. H. Muller, K. Mader, S. Gohla, "Solid lipid nanoparticle (SLN) for controlled drug delivery-a review of the state of the art", Eur. J. Pharm. Biopharm., Vol. 50, (2000), pp. 161-177.

[16] E. N. Dolatabali, H. Hamishehkar, M. Eskandani, H. Valizadeh, "Formulation, characterization and cytotoxicity studies of alendronate sodium-loaded solid lipid nanoparticle", Colloid Surf. B. Biointerfaces., Vol. 117, (2014), pp. 21-28.

[17] R, M. Shah, F. Malherbe, D. Eldridge, E. A. Palombo, I. H. Harding, "Physiochemical characterization of solid lipid nanoparticle (SLN) prepared by a novel micro-emulsion technique" J. Colloid. Interface., Vol. 428, (2014), pp. 286-294.

[18] X. Yang, Y. Liu, C. Liu, N. Zhang, "Biodegradable solid lipid nanoparticle flocculates for pulmonary delivery of insulin", J. Biomed. Nanotechnol., Vol. 8, (2005), pp. 834-842.

[19] H. Li, X. Zhao, Y. Ma, G. Zhai, L. Li, H. Lou, "Enhancement of gastrointestinal absorption of quercetin by solid lipid nanoparticle”, J. Control. Release., Vol. 133, (2009), pp. 238-244.

[20] S. Jose, S. S. Anju, T. A. Cinu, N. A. Aleykutty, S. Thomas, E. B. Souto, "In vivo pharmacokinetics and biodistribution of resvetarol-loaded solid lipid nanoparticle for brain delivery”, Int. J. Pharm., Vol. 474, (2014), pp. 6-13.

[21] L. Ambarsari, W. Nurcholis, L. K. Darusman, M A. Mujib, R. Heryanto, "The curcuminoids extract of curcuma xanthorriza roxb loaded solid lipid nanoparticles", Int. J. Sci. Res., Vol. 3, (2014), pp. 852-856.

[22] B. B. Aggarwal, S. Shishodia, Y. Takada, "Curcumin suppresses the paclitaxel-induced nuclear factor$\mathrm{kB}$ pathway in breast cancer cells and inhibits lung metastasis of human breast cancer in nude mice", Clin. Cancer Res., Vol. 11, (2005), pp. 7490-7498.

[23] K. Raparia, C. Villa, M. M. Decamp, J. D. Patel, M. P. Mehta, "Molecular profiling in non-small cell lung cancer: a step toward personalized medicine", Arch. Pathol. Lab. Med., Vol. 137, (2013), pp. 481491.

[24] P M. Green, S. Guerrier-Adams, P. O. Okunji, D. Schiavone, J. E. Smith, "African American health disparities in lung cancer”, Clin. J. Oncol. Nurs., Vol. 17, (2013), pp.180-186.

[25] M. Pradita, "Aktifitas antiinflamasi sediaan nanopartikel ekstrak kurkuminoid temulawak tersalut asam palmitat secara in vivo", Bogor Agricultural University, Bogor, (2014).

[26] S. Mukherjee, S. Ray, R. S. Takur, "Solid lipid nanoparticle: a modern formulation approach in drug delivery system”, Indian J. Pharm. Sci., Vol. 71, (2009), pp. 349-358.

[27] C. Schwarz, W. Mehnert, J. S.Lucks, R. H. Muller, "Solid lipid nanoparticle (SLN) for controlled drug delivery, I, production, characterization and sterilization”, J. Control Release., Vol. 30, (1994), pp. 8396.

[28] S. K. Sahoo, V. Labhasetwar, "Enhanced anti-proliferativ activity of transferrin-conjugated paclitaxelloaded nanoparticle is mediated via sustained intracellular drug retention", Mol. Pharm., Vol. 22, (2005), pp. 373-383

[29] J. Hao, X. Fang, Y. Zhao, "Development and optimization of solid lipid nanoparticle formulation for ophthalmic delivery of chloramphenicol using a box-behnken design", Int. J. Nanomedicine., Vol. 4, (2011), pp. 683-692.

[30] K. Dilen, W. Weyenberg, J. Vandervoort, A. Ludwig, "The influence of the use of viscosifying agents as dispertion media on the drug release properties from PLGA nanoparticles", Eur J Pharm Biopharm., Vol. 58, (2004), pp. 539-549.

[31] A. Mitra, S. Lin, "Effect of surfactant on fabrication and characterization of paclitaxel-loaded polybutylcyanocrylate nanoparticulate delivery systems", J. Pharm. Pharmacol., Vol. 55, (2003), pp. 895-902.

[32] M. Wiśniewska, "The temperature effect on electro kinetic properties of the silica-polyvinyl alcohol (PVA) system”, Colloid Polym. Sci., Vol. 289, (2011), pp. 341-344. 
[33] M. Uner, G. Yener, "Importance of SLN in various administration routes and future perspective. Int. J. Nanomed., Vol. 2, (2007), pp. 289.

[34] N. Aggarwal, S. Goindi, "Preparation and in vivo evaluation of solid lipid nanoparticles: preparation, characterization and antitumor activity, cellular uptake and improve in vivo bioavailability", J. Biomed. Nanotechol., Vol. 4, (2013), pp. 564-576.

[35] T. Mosmann, "Rapid colorimetric assay for cellular growth and survival: Application to proliferation and cytotoxicity assays", J. Immunol. Methods., Vol. 65, (1983), pp. 55-63.

[36] R. Abbasalipourkabir, A. Salehzadeh, R. Abdullah, "Cytotoxicity effect of solid lipid nanoparticles on human breast cancer cell lines", Biotech., Vol. 10, (2011), pp. 528-533.

[37] S. Rohit, J. Monkkonen, O. Risto, R. Kakasaheb, R. P. Anant, "Transferrin mediated solid lipid nanoparticle containning curcumin: Enhanced in vitro anticancer activity by induction of apoptosis", Int. J. Pharm., Vol. 389, (2010), pp. 190-200.

[38] J. Ye, Q. Wang, X. Zhoua, N. Zhang, "Injectable loaded solid lipid nanoparticles as passive targeting therapeutic agents for rheumatoid arthritis", Int. J. Pharm., Vol. 352, (2008) pp. 273-279.

[39] L. H. Reddy, K. Vivek, N. Bakshi, R. S. Murthy, "Tamoxifen citrate loaded solid lipid nanoparticles (SLN): preparation, characterization, in vitro drug release, and pharmacokinetic evaluation", Pharm. Dev. Technol., Vol. 11, (2006), pp.167-177. 